\title{
LESÃO RENAL AGUDA EM UNIDADE DE TRATAMENTO INTENSIVO: CARACTERÍSTICAS CLÍNICAS E DESFECHOS
}

\author{
Jaqueline Luft ${ }^{1}$, Adilson Adair Boes ${ }^{2}$, Daniele Delacanal Lazzari ${ }^{3}$, Eliane Regina Pereira do Nascimento ${ }^{4}$, \\ Juliano de Amorim Busana ${ }^{5}$, Bruna Pedroso Canever ${ }^{3}$
}

\begin{abstract}
RESUMO: Estudo quantitativo, documental, retrospectivo, que objetivou caracterizar o perfil clínicodemográfico e o desfecho de pacientes com insuficiência renal, submetidos à terapia dialítica em uma unidade de terapia intensiva adulto de um hospital geral da região sul do Brasil. Os dados foram coletados no período de janeiro a fevereiro de 2013 e a amostra, constituída por 74 prontuários. Identificou-se prevalência do sexo masculino, média de $63,43 \pm 14,73$ anos, o motivo clínico foi o mais frequente para internação, com uma ou mais comorbidades associadas. A lesão renal com maior prevalência foi a crônica agudizada, pré-renal. Com relação aos motivos que levaram à lesão, o choque séptico foi mais recorrente. O tratamento de escolha foi hemodiálise convencional, por cateter. O desfecho mais comum foi o óbito. Desta forma, a identificação de fatores de risco e conhecimento dos fatores associados fornece subsídios para rápida identificação de problemas e implementação de ações de enfermagem.
\end{abstract}

DESCRITORES: Pacientes internados; Insuficiência renal; Terapia intensiva; Diálise renal.

\section{CHRONIC KIDNEY INJURY AT AN INTENSIVE CARE SERVICE: CLINICAL CHARACTERISTICS AND OUTCOMES}

ABSTRACT: The objective in this quantitative and retrospective documentary study was to characterize the clinical-demographic profile and outcome of kidney failure patients submitted to dialysis therapy at an adult intensive care unit of a general hospital in the South of Brazil. The data were collected between January and February 2013 and the sample consisted of 74 patient histories. The prevalence of men was identified, with a mean age of $63.43 \pm 14.73$ years, clinical motives were the most frequent cause of hospitalization and one or more associated comorbidities. The most prevalent kidney injury was acute chronic, pre-renal injury. The most recurrent motive for the injury was septic shock. The preferred treatment was conventional hemodialysis using a catheter. The most common outcome was death. The, the identification of risk factors and knowledge about the associated factors supports the rapid identification of problems and the implementation of nursing actions.

DESCRIPTORS: Inpatients; Renal insufficiency; Critical care; Renal dialysis.

\section{LESIÓN RENAL AGUDA EN UNIDAD DE TRATAMIENTO INTENSIVO: CARACTERÍSTICAS CLÍNICAS Y DESFECHOS}

RESUMEN: Estudio cuantitativo, documental, retrospectivo, con objeto de caracterizar el perfil clínico-demográfico y el desfecho de pacientes con insuficiencia renal, sometidos a la terapia dialítica en una unidad de terapia intensiva adulto de un hospital general de la región sur de Brasil. Los datos fueron recolectados en enero y febrero del 2013 y la muestra incluyó a 74 archivos. Fue identificada prevalencia del sexo masculino, edad media $63,43 \pm 14,73$ años, el motivo clínico fue el más frecuente para internación, con una o más comorbilidades asociadas. La lesión renal con mayor prevalencia fue la crónica agudizada, pre-renal. Respecto a los motivos de la lesión, el choque séptico fue el más recurrente. El desfecho más común fue el óbito. Así, la identificación de factores de riesgo y el conocimiento de los factores asociadosapoyan la rápida identificación de problemas y la implementación de acciones de enfermería. DESCRIPTORES: Pacientes internos; Insuficiencia renal; Cuidados críticos; Diálisis renal.

${ }^{1}$ Enfermeira. Enfermeira assistencial. Hospital Mãe de Deus (Porto Alegre). Porto Alegre, RS, Brasil.

${ }^{2}$ Enfermeiro. Mestre em Biologia Celular e Molecular aplicada à saúde. Docente de Enfermagem da Universidade Feevale. Novo Hamburgo, RS, Brasil.

${ }^{3}$ Enfermeira. Doutora em Enfermagem. Universidade Federal de Santa Catarina. Florianópolis, SC, Brasil.

${ }^{4}$ Enfermeira. Doutora em Enfermagem. Docente de Enfermagem da Universidade Federal de Santa Catarina. Florianópolis, SC, Brasil.

${ }^{5}$ Enfermeiro. Mestre em Enfermagem. Universidade Federal de Santa Catarina. Florianópolis, SC, Brasil. 


\section{- INTRODUÇÃO}

O termo Lesão Renal Aguda (LRA) é utilizado para refletir todo o espectro de Insuficiência Renal Aguda (IRA), reconhecendo que um declínio agudo da função renal é frequentemente secundário a uma lesão que provoca alterações funcionais ou estruturais nos rins ${ }^{(1)}$. Diante dos avanços tecnológicos, do aumento da sobrevida da população e da sofisticação da terapêutica, a LRA ainda é uma das complicações mais frequentes encontradas na Unidade de Terapia Intensiva (UTI). A associação dos fatores de risco, evolução clínica e as múltiplas intervenções no paciente na UTI contribuem para a manutenção das taxas elevadas de morbidade e mortalidade da LRA, sem melhora significativa nestas taxas há, pelo menos, duas décadas ${ }^{(2-3)}$.

A LRA em pacientes hospitalizados é caracterizada pela redução abrupta da Taxa de Filtração Glomerular (TFG) que se mantém por períodos variáveis de tempo, tendo como resultado, a inabilidade dos rins para exercer as funções de excreção, manter o equilíbrio ácido-básico e homeostase hidroeletrolítica ${ }^{(4)}$. As complicações da LRA são responsáveis por contribuir significativamente para a elevação das taxas de morbidade de pacientes em estado crítico $^{(5)}$. Apesar de seu caráter potencialmente catastrófico, a LRA é, em princípio, reversível e há chances de recuperação sem alterações de outros órgãos ${ }^{(3,6)}$.

Estudos $^{(3,7)}$ revelam que a presença de condições que determinam hipoperfusão e isquemia renal, está relacionada diretamente com o desenvolvimento da LRA e os pacientes que apresentam redução da reserva funcional renal são mais suscetíveis a desenvolver tal complicação, mesmo com lesões renais pequenas. Idade avançada, presença de Diabetes Mellitus, de Hipertensão Arterial e de Insuficiência Cardíaca Congestiva, além do uso crônico de anti-inflamatórios não hormonais, são considerados fatores de risco para a $\mathrm{LRA}^{(7)}$.

O desenvolvimento de complicações durante a internação em uma UTI, tais como infecções, sepse, hemorragias, cirurgias e necessidade de diálise, pode fazer com que o nível de severidade do paciente e da LRA seja maior. Em pacientes hospitalizados, a LRA é considerada uma das complicações mais importantes, sendo que sua incidência varia de acordo com as condições clínicas dos pacientes, ocorrendo em maior quantidade em UTIs (20 a 40\%) e em menor quantidade em unidades intermediárias $(1 \text { a } 7 \%)^{(8-9)}$.

Fatores diversos podem contribuir para taxa elevada de mortalidade em LRA, particularmente a não identificação de fatores de risco, diagnóstico tardio da doença ou desconhecimento de fatores associados à mortalidade. A incidência de pacientes internados em UTI que evoluem para LRA varia entre $17 \%$ a $35 \%$, sendo que $49 \%$ a $70 \%$ necessitam de tratamento dialítico. A mortalidade por LRA nas UTIs varia entre $50 \%$ a $90 \%$ e está associada ao tempo de internação prolongado, uso de terapias com tecnologias avançadas, tipo de UTI e população estudada, sendo a LRA a complicação mais frequentemente encontrada em pacientes em UTI ${ }^{(9)}$. Em função das complicações associadas e das condições clínicas do paciente em terapia intensiva, além da não melhora significativa mesmo diante dos esforços contínuos em busca da terapêutica ideal, deve-se considerar o subdiagnóstico ou diagnóstico tardio como fatores contribuintes para a alta mortalidade ${ }^{(8)}$.

Neste contexto, o objetivo deste estudo foi caracterizar o perfil clínico-demográfico e o desfecho de pacientes com insuficiência renal, submetidos à terapia dialítica em uma unidade de terapia intensiva adulto.

\section{MÉTODO}

Estudo quantitativo, com delineamento transversal, de caráter documental e retrospectivo em uma Unidade de Terapia Intensiva adulto. A amostra foi por conveniência e composta por 74 prontuários, sendo considerados como critérios de inclusão: pacientes com idade superior a 18 anos, diagnóstico de Insuficiência Renal, submetidos à terapia dialítica durante os meses de janeiro de 2011 a dezembro de 2012, os registros deveriam estar completos.

Foram excluídos do estudo oito prontuários de pacientes que evoluíram para IRA, porém não 
necessitaram de terapia dialítica, quatro prontuários de pacientes com insuficiência renal crônica não agudizada, três prontuários ilegíveis e um com registro inadequado, totalizando 16 prontuários excluídos.

Para a coleta de dados, foi elaborado um instrumento de pesquisa contendo informações de identificação do perfil demográfico e clínico dos pacientes, destacando a idade, sexo, comorbidades prévias, tipo de hemodiálise realizada e desfecho.

Os dados foram coletados no período de janeiro a fevereiro de 2013, e transcritos para o banco de dados em planilha do Microsoft Office Excel para ser submetido ao processo de análise descritiva. Os resultados das variáveis nominais foram expressos por análises de frequência e os resultados das variáveis contínuas, expressos pela média \pm desvio padrão.

Para verificar a associação entre o desfecho e as demais variáveis nominais, foi utilizado Quiquadrado ou Teste Exato de Fisher de acordo com as suposições dos testes. Para verificar a associação entre as variáveis contínuas, foi utilizada a Análise de Variância One-Way ou teste t, de acordo com as suposições dos testes. Para verificar a associação com as demais variáveis, foi utilizado o teste Quiquadrado. Para verificar a normalidade dos dados, foi utilizado o teste Kolmogorov-Smirnov. Para todas as variáveis foi considerado significativo um $p<0,05$.

O projeto foi aprovado em 25 de fevereiro de 2013 pelo Comitê de Ética e Pesquisa da Federação de Estabelecimento de Ensino Superior em Novo Hamburgo - Feevale, sob parecer número 184-947.

\section{- RESULTADOS}

Os resultados obtidos demonstram a predominância o sexo masculino em pacientes acometidos por insuficiência renal, com idades entre 23 a 86 anos, com idade média de 63,43 \pm 14,73 anos, sendo o motivo mais frequente da internação aquele relacionado a questões clínicas e à existência de uma ou mais comorbidades associadas, conforme a Tabela 1.

Dentre as comorbidades encontradas no estudo com menor frequência registrou-se: obesidade, vasculopatias periférica, hepatite $\mathrm{C}$, tabagismo, artrite reumatoide, colicistite aguda, varizes esofágicas, dislipidemia, depressão, osteomielite, hematoma subdural, endocardite, hipotireoidismo, infarto agudo do miocárdio, intoxicação exógena, doença de Parkinson, retocolite ulcerativa crônica, rins policísticos e Síndrome de Sjögren.

Com relação aos dados da insuficiência renal, verificou-se que 54 pacientes (73\%) possuíam Insuficiência Renal Crônica agudizada pré-renal. Analisou-se a frequência de uma ou mais enfermidades durante a internação em UTI, sendo a de maior ocorrência a sepse, seguida de problemas respiratórios, insuficiência renal e o choque séptico; a oscilação dos valores da creatinina sérica em diferentes momentos; e o volume urinário, de acordo com a Tabela 2.

A Tabela 3 apresenta as principais causas da LRA na UTI, com predominância do choque séptico, seguido pela acidose metabólica e a sepse. Com relação à modalidade de hemodiálise, predominou o método contínuo; o tipo de anticoagulação mais frequente foi a regional, sendo que em 17 (22,9\%) pacientes não foi utilizado nenhum anticoagulante, apenas salinização do sistema. Neste último dado, os resultados na tabela totalizaram soma superior a 100\%, em função de alguns pacientes terem utilizado mais de um tipo de anticoagulante durante sua internação, desta forma, constam na Tabela 3, 87 registros $(117,5 \%)$ com relação à anticoagulação. O acesso para diálise com maior predominância foi o cateter para hemodiálise.

Alguns pacientes foram submetidos aos dois tipos de hemodiálise citados anteriormente, sendo a soma destes (95) (128,38\%) maior que o n (74 - 100\%). Não foi registrada diálise peritoneal no período da coleta.

Quanto ao desfecho dos pacientes submetidos à terapia dialítica na UTI, evidenciou-se a predominância de óbito (54 pacientes) (72,9\%), 17 (22,9\%) pacientes receberam alta para a unidade de internação e três foram transferidos para outro hospital. Ao analisar a causa dos óbitos, o principal motivo foi a disfunção de múltiplos órgãos, seguido pelo choque séptico e sepse. 
Tabela 1 - Perfil dos pacientes e características do momento da internação. Novo Hamburgo, RS, Brasil, 2013

\begin{tabular}{|c|c|c|}
\hline Variáveis & $\mathbf{n}$ & $\%$ \\
\hline \multicolumn{3}{|l|}{ Sexo } \\
\hline Masculino & 46 & 62,2 \\
\hline Feminino & 28 & 37,8 \\
\hline Idade (a) & \multicolumn{2}{|c|}{$63,43(\mathrm{dp} \mathrm{14,73)}$} \\
\hline \multicolumn{3}{|l|}{ Raça } \\
\hline Branco & 36 & 48,6 \\
\hline Pardo & 3 & 4,1 \\
\hline Não identificado no prontuário & 35 & 47,3 \\
\hline \multicolumn{3}{|l|}{ Motivo da internação } \\
\hline Clínico & 56 & 75,7 \\
\hline Cirúrgico & 18 & 24,3 \\
\hline \multicolumn{3}{|l|}{ Local de origem } \\
\hline Emergência & 21 & 28,4 \\
\hline Unidade de internação & 43 & 58,1 \\
\hline Outros & 10 & 13,5 \\
\hline \multicolumn{3}{|l|}{ Comorbidades encontradas } \\
\hline Problemas respiratórios & 44 & 59,46 \\
\hline $\begin{array}{l}\text { Hipertensão arterial sistêmica } \\
\text { (HAS) }\end{array}$ & 33 & 44,59 \\
\hline Diabetes Mellitus & 22 & 29,73 \\
\hline Cardiopatias & 19 & 25,68 \\
\hline Neoplasias & 16 & 21,62 \\
\hline Acidente vascular encefálico & 12 & 16,22 \\
\hline Cirrose hepática & 7 & 9,46 \\
\hline Choque séptico & 6 & 8,11 \\
\hline
\end{tabular}

Tabela 2 - Dados sobre Lesão Renal Aguda durante a internação do paciente. Novo Hamburgo, RS, Brasil, 2013

\begin{tabular}{|c|c|c|c|}
\hline \multicolumn{2}{|l|}{ Variáveis } & $\mathbf{N}$ & $\%$ \\
\hline \multicolumn{4}{|l|}{ Tipo de lesão renal } \\
\hline \multicolumn{2}{|l|}{ Aguda } & 20 & 27 \\
\hline \multicolumn{2}{|l|}{ Crônica agudizada } & 54 & 73 \\
\hline \multicolumn{4}{|c|}{ Enfermidades durante a internação } \\
\hline \multicolumn{2}{|l|}{ Sepse } & 34 & 45,95 \\
\hline \multicolumn{2}{|l|}{ Problemas respiratórios } & 14 & 18,92 \\
\hline \multicolumn{2}{|l|}{ Insuficiência renal aguda } & 13 & 17,57 \\
\hline \multicolumn{2}{|l|}{ Choque Séptico } & 6 & 8,11 \\
\hline \multicolumn{2}{|l|}{ Choque cardiogênico } & 3 & 4,05 \\
\hline \multicolumn{2}{|l|}{ Encefalopatia } & 2 & 2,7 \\
\hline \multicolumn{2}{|l|}{ Endocardite } & 2 & 2,7 \\
\hline \multicolumn{2}{|c|}{ Infarto agudo do miocárdio } & 2 & 2,7 \\
\hline \multicolumn{2}{|c|}{ Parada cardiorrespiratória } & 2 & 2,7 \\
\hline \multicolumn{2}{|l|}{ Choque hipovolêmico } & 1 & 1,35 \\
\hline \multicolumn{2}{|c|}{ Derrame pericárdico } & 1 & 1,35 \\
\hline \multicolumn{2}{|c|}{ Hematoma de parede abdominal } & 1 & 1,35 \\
\hline \multicolumn{2}{|c|}{ Hipotensão } & 1 & 1,35 \\
\hline \multicolumn{2}{|l|}{ Insuficiência hepática } & 1 & 1,35 \\
\hline \multicolumn{2}{|l|}{ Mediastinite } & 1 & 1,35 \\
\hline \multicolumn{4}{|l|}{ Volume urinário } \\
\hline \multicolumn{2}{|l|}{ Anúria } & 22 & 29,7 \\
\hline \multicolumn{2}{|l|}{ Oligúria } & 52 & 70,3 \\
\hline \multicolumn{4}{|c|}{ Classificação da lesão renal aguda } \\
\hline \multicolumn{2}{|l|}{ Pré-Renal } & 61 & 82,4 \\
\hline \multicolumn{2}{|l|}{ Renal } & 6 & 8,1 \\
\hline \multicolumn{2}{|l|}{ Pós Renal } & 7 & 9,5 \\
\hline Valor da creatinina & Mínimo & Máximo & Média \\
\hline $\begin{array}{l}\text { No momento da } \\
\text { internação }\end{array}$ & 0,4 & 7,6 & $\begin{array}{c}2,62 \pm \\
1,91 \\
\end{array}$ \\
\hline $\begin{array}{l}\text { No momento } \\
\text { da indicação de } \\
\text { hemodiálise }\end{array}$ & 0,98 & 13,06 & $\begin{array}{c}4,09 \pm \\
2,15\end{array}$ \\
\hline $\begin{array}{l}\text { No momento do início } \\
\text { da hemodiálise }\end{array}$ & 0,98 & 13,06 & $\begin{array}{c}4,30 \pm \\
2,19 \\
\end{array}$ \\
\hline $\begin{array}{l}\text { Ao término do } \\
\text { tratamento }\end{array}$ & 0,58 & 9,2 & $\begin{array}{c}3,02 \pm \\
1,81\end{array}$ \\
\hline
\end{tabular}

Na Tabela 5 apresenta-se a associação entre o desfecho e as demais variáveis do estudo. Verificou-se que $33(61,11 \%)$ dos pacientes que evoluíram para óbito eram do sexo masculino, com idade 65,26 \pm 13,23 anos; da raça branca, com motivo de internação clínica, encaminhados para a UTI procedentes da unidade de internação, portadores de IRA pré-renal, com valores de creatinina sérica maior que 3mg/ $\mathrm{dL}$, em uso de cateter para hemodiálise e que necessitaram de terapia vasopressora.

Para as variáveis demográficas, observou-se que em relação à idade, os pacientes que evoluíram para óbito possuíam média superior $(65,26 \pm 13,23$ anos) quando comparados aos que receberam alta para a unidade (59 $\pm 18,06$ anos). Não houve diferença estatisticamente significa entre o óbito e sexo dos pacientes $(p>0,38)$. 
Tabela 3 - Causas que levaram à Lesão Renal Aguda e informações sobre a hemodiálise. Novo Hamburgo, RS, Brasil, 2013

\begin{tabular}{|c|c|c|}
\hline Variáveis & $\mathbf{n}$ & $\%$ \\
\hline \multicolumn{3}{|l|}{ Motivo da LRA } \\
\hline Choque séptico & 51 & 68,92 \\
\hline Acidose metabólica & 5 & 6,76 \\
\hline Sepse & 5 & 6,76 \\
\hline Problemas respiratórios & 3 & 4,05 \\
\hline Hipercalemia & 3 & 4,05 \\
\hline Mediastinite & 2 & 2,7 \\
\hline Necrose tubular aguda & 2 & 2,7 \\
\hline Parada Cardiorrespiratória & 2 & 2,7 \\
\hline Baixo débito cardíaco & 1 & 1,35 \\
\hline Neoplasia & 1 & 1,35 \\
\hline $\begin{array}{l}\text { Insuficiência Renal Aguda } \\
\text { hepatorenal }\end{array}$ & 1 & 1,35 \\
\hline $\begin{array}{l}\text { Insuficiência Renal Aguda } \\
\text { multifatorial }\end{array}$ & 1 & 1,35 \\
\hline Uremia & 1 & 1,35 \\
\hline \multicolumn{3}{|l|}{ Método de hemodiálise } \\
\hline Intermitente & 39 & 52,7 \\
\hline Convencional & 56 & 75,68 \\
\hline \multicolumn{3}{|l|}{ Anticoagulante } \\
\hline Sistêmica & 8 & 10,81 \\
\hline Regional & 62 & 83,78 \\
\hline Sem anticoagulação & 17 & 22,97 \\
\hline \multicolumn{3}{|l|}{ Acesso para diálise } \\
\hline Fístula arteriovenosa & 5 & 6,8 \\
\hline Cateter para hemodiálise & 69 & 93,2 \\
\hline Tempo de diálise em dias & \multicolumn{2}{|c|}{$6,36 \pm 6,61$} \\
\hline $\begin{array}{l}\text { Tempo de internação na UTI em } \\
\text { dias }\end{array}$ & \multicolumn{2}{|c|}{$18,76 \pm 2,63$} \\
\hline $\begin{array}{l}\text { Tempo na unidade de internação } \\
\text { em dias }\end{array}$ & \multicolumn{2}{|c|}{$36,17 \pm 29,90$} \\
\hline \multicolumn{3}{|l|}{ Uso de vasopressor } \\
\hline Sim & 65 & 87,8 \\
\hline Não & 9 & 2,12 \\
\hline
\end{tabular}

Tabela 4 - Desfecho dos pacientes em terapia dialítica na UTI. Novo Hamburgo, RS, Brasil, 2013

\begin{tabular}{lcc} 
Variáveis & $\mathbf{n}$ & $\mathbf{\%}$ \\
\hline Desfecho dos pacientes & \multicolumn{2}{c}{} \\
\hline Alta para a unidade de internação & 17 & 23 \\
\hline Transferência para outro hospital & 3 & 4 \\
\hline Óbito & 54 & 73 \\
\hline Desfecho dos pacientes que receberam altas \\
\hline Necessitaram de hemodiálise & 4 & 23,52 \\
\hline Não necessitaram de hemodiálise & 13 & 76,48 \\
\hline Causa do óbito & & \\
\hline Disfunção de múltiplos órgãos & 34 & 62,96 \\
\hline Choque séptico & 10 & 18,52 \\
\hline Sepse & 5 & 9,25 \\
\hline Parada cardiorrespiratória & 2 & 3,7 \\
\hline Acidente vascular encefálico & 1 & 1,85 \\
hemorrágico & \multicolumn{1}{l}{} \\
\hline Choque cardiogênico & 1 & 1,85 \\
\hline Insuficiência cardíaca & 1 & 1,85
\end{tabular}

Em relação aos valores de creatinina sérica no término do tratamento, seja por alta para a unidade ou que evoluíram a óbito, houve diferença significativamente estatística $(p<0,01)$. O tempo de diálise em dias em relação aos pacientes que receberam alta para a unidade de internação $(9,88 \pm 8,11)$ e os pacientes que foram a óbito $(54 \pm 5,44)$ foi estatisticamente maior em relação aos que faleceram $(p<0,04)$. Os pacientes que evoluíram para óbito fizeram uso de terapia vasopressora $(p<0,04)$.

\section{DISCUSSÃO}

A predominância do sexo masculino $(62,2 \%)$ entre os pacientes que desenvolveram LRA na UTI corrobora com outros estudos que encontraram resultado semelhante, ainda que a diferença entre os sexos seja discreta. Outros dois estudos ${ }^{(2,10)}$ tiveram por objetivo caracterizar os pacientes portadores de insuficiência renal aguda (IRA) e apontaram prevalências de 55,7\% e 72\%, respectivamente, de pacientes do sexo masculino.

Com relação à idade, estudo ${ }^{(10)}$ realizado em Joaçaba, estado de Santa Catarina, com o objetivo de identificar o perfil dos pacientes com IRA, internados na unidade de terapia intensiva, apresentou média oscilando entre 60 e 70 anos, sendo que frequência da ocorrência de IRA foi diretamente proporcional 
Tabela 5 - Associação entre o desfecho de pacientes com insuficiência renal em terapia dialítica com as demais variáveis de estudo. Novo Hamburgo, RS, Brasil, 2013

\begin{tabular}{|c|c|c|c|c|}
\hline & \multicolumn{4}{|c|}{ Desfecho } \\
\hline & $\begin{array}{c}\text { Alta para a } \\
\text { unidade } n=17\end{array}$ & $\begin{array}{c}\text { Transferência } \\
n=3\end{array}$ & $\begin{array}{l}\text { Óbito } \\
\mathrm{n}=54\end{array}$ & $P$ \\
\hline Sexo & & & & 0,38 \\
\hline Masculino & $10(58,82 \%)$ & $3(100 \%)$ & $33(61,11 \%)$ & \\
\hline Feminino & $7(41,185)$ & $0(0 \%)$ & $21(38,895)$ & \\
\hline Idade & $59 \pm 18,06$ & $55,33 \pm 13,32$ & $65,26 \pm 13,23$ & 0,2 \\
\hline \multicolumn{5}{|l|}{ Raça } \\
\hline Branco & $10(58,82 \%)$ & $0(0 \%)$ & $26(48,15 \%)$ & 0,3 \\
\hline Pardo & $0(05)$ & $0(0 \%)$ & $3(56,56 \%)$ & \\
\hline Outras & $7(41,18 \%)$ & $3(100 \%)$ & $25(46,30 \%)$ & \\
\hline Motivo da internação & & & & 0,1 \\
\hline Clinico & $15(88,24 \%)$ & $1(33,33 \%)$ & $40(74,07 \%)$ & \\
\hline Cirúrgico & $2(11,76 \%)$ & $2(66,67 \%)$ & $14(25,93 \%)$ & \\
\hline Local de origem & & & & 0,16 \\
\hline Emergência & $9(52,94 \%)$ & $1(33,33 \%)$ & $11(20,37 \%)$ & \\
\hline Unidade de internação & $6(35,29 \%)$ & $1(33,33 \%)$ & $34(62,96 \%)$ & \\
\hline Outra UTI & $0(0 \%)$ & $0(0 \%)$ & $3(5,56 \%)$ & \\
\hline Outros & $2(11,76 \%)$ & $1(33,33 \%)$ & $6(11,11 \%)$ & \\
\hline Insuficiência renal & & & & 0,42 \\
\hline Aguda & $11(64,71 \%)$ & $3(100 \%)$ & $40(74,07 \%)$ & \\
\hline Crônica agudizada & $6(35,295)$ & $0(0 \%)$ & $14(25,93 \%)$ & \\
\hline Classificação IRA & & & & 0,36 \\
\hline Pré-renal & $15(88,24 \%)$ & $2(66,67 \%)$ & $44(81,48 \%)$ & \\
\hline Renal & $2(11,76 \%)$ & $0(0 \%)$ & $4(7,41 \%)$ & \\
\hline Pós-renal & $0(0 \%)$ & $0(0 \%)$ & $6(11,11 \%)$ & \\
\hline \multicolumn{5}{|l|}{ Valor da creatinina } \\
\hline Na internação (a) & $3,18 \pm 2,32$ & $3,11 \pm 2,82$ & $2,40 \pm 1,72$ & 0,31 \\
\hline $\mathrm{Na}$ indicação (a) & $4,83 \pm 1,92$ & $4,57 \pm 2,71$ & $3,82 \pm 2,17$ & 0,27 \\
\hline Na hemodiálise (a) & $5,18 \pm 2,04$ & $4,57 \pm 2,71$ & $3,82 \pm 2,17$ & 0,12 \\
\hline No término do tratamento (a) & $2,22 \pm 1,76$ & $5,45 \pm 1,34$ & $3,14 \pm 1,72$ & 0,01 \\
\hline Tempo de diálise (dias) & $9,88 \pm 1,76$ & $6 \pm 3,61$ & $54 \pm 5,44$ & 0,04 \\
\hline Acesso para diálise & & & & 0,6 \\
\hline Sim & $2(11,76 \%)$ & $0(0 \%)$ & $3(5,56 \%)$ & \\
\hline Não & $15(88,24 \%)$ & $3(100 \%)$ & $51(94,44 \%)$ & \\
\hline Uso de vasopressor & & & & 0,04 \\
\hline Sim & $11(64,71 \%)$ & $3(100 \%)$ & $51(94,44 \%)$ & \\
\hline Não & $6(35,295)$ & $0(0 \%)$ & $3(5,56 \%)$ & \\
\hline
\end{tabular}

à faixa etária dos pacientes. Em estudo ${ }^{(11)}$ realizado para avaliar as complicações de procedimentos hemodialíticos em 65 pacientes com LRA, a faixa etária variou entre $60,9 \pm 16,2$ anos, sendo que 32 pacientes $(49,2 \%)$ possuíam idade igual ou superior a 65 anos. Este mesmo estudo encontrou ainda o predomínio da raça branca $(93,8 \%)$. Esta informação corrobora com as encontradas neste estudo, em que $48,6 \%$ dos pacientes eram da raça branca, ressaltando as características regionais de predominância da colonização alemã e italiana. Dos prontuários que compuseram esta pesquisa, 47,3\% não possuíam a especificação da raça. 
Sobre o motivo da internação, em $75,7 \%$ dos pacientes esta decorreu de complicações clínicas, e outros $24,3 \%$ tiveram motivo cirúrgico. Estes dados vão ao encontro de estudo(8) que verificou que parte dos pacientes que evoluíram para LRA foi internada por uma causa clínica, seguida de causa cirúrgica.

As comorbidades mais encontradas foram os problemas respiratórios (59,46\%). O desenvolvimento de complicações pulmonares, tais como edema, derrame pleural e infecções, são frequentes em pacientes com doença renal, devido a alterações mecânicas e hemodinâmicas. O surgimento de edema pulmonar bilateral tem relação com o aumento da volemia e elevados valores séricos de substâncias osmoticamente ativas ${ }^{(12)}$.

Em estudo ${ }^{(13)}$ que observou o desfecho de pacientes com câncer internados em UTI com LRA, a comorbidade mais prevalente foi a HAS. Os resultados apresentados comprovam que a existência de comorbidades caracterizadas como crônicas, constituem fatores de predisposição para o desenvolvimento de LRA, sendo que os pacientes com estas enfermidades prévias devem ser monitorados com maior atenção, buscando a implementação de medidas protetoras e acompanhamento para o diagnóstico e tratamento precoce da LRA.

A ocorrência de LRA em pacientes criticamente enfermos é significativa em todo o mundo, incluindo os países desenvolvidos, alertando para a necessidade dos profissionais de saúde aprofundar seus conhecimentos sobre a terapêutica e o tratamento precoce, otimizando o uso de drogas nefrotóxicas, por exemplo, dentre outras inúmeras condutas ${ }^{(14-15)}$.

Neste estudo verificou-se que $73 \%$ dos pacientes apresentou IR crônica agudizada, enquanto que os demais desenvolveram IR aguda. A LRA pode ser classificada em pré-renal, renal e pós-renal, e os resultados na UTI pesquisada revelaram causa pré-renal em $82,4 \%$ dos pacientes. Esta é a etiologia mais comum em pacientes com LRA, e é assim denominada por provocar a hipoperfusão renal, sem comprometimento da estrutura do parênquima renal ${ }^{(16)}$. Casos de LRA pré-renal podem ser revertidos no primeiro ou segundo dias de instalação da doença, porém mediante tratamento adequado ${ }^{(15-16)}$. A predominância de LRA pré-renal tem relação com o volume urinário, sendo que a oligúria, condição em que os rins mantém um nível de atividade de filtração menor que a necessária para manter a homeostase, ocorreu em $70,3 \%$ dos pacientes.

Quanto às patologias desenvolvidas durante a internação, 45,95\% dos pacientes desta pesquisa apresentaram sepse, seguida de problemas respiratórios (18,92\%) e LRA (17,57\%). O diagnóstico de sepse é frequentemente apontado por outros estudos, inclusive como uma das causas principais de prognóstico reservado ${ }^{(17)}$.

Os valores de creatinina sérica são de extrema importância para o diagnóstico e acompanhamento da evolução da LRA. Para esta pesquisa, a média no momento da internação foi de 2,62 ml/dl, no momento da indicação foi de 4,09 ml/dl, no início da hemodiálise de 4,30 ml/dl e ao final do tratamento, 3,02 ml/ dl. A creatinina sérica é um importante marcador do estado nutricional e apresenta relações com a mortalidade de pacientes com enfermidades renais em terapia intensiva ${ }^{(18)}$. A creatinina manteve-se com valores acima dos considerados de referência em todos os exames. O aumento entre o primeiro e o quarto exame reflete a acumulação de substâncias que, normalmente, seriam removidas pela filtração glomerular.

Cabe ressaltar que a creatinina é um marcador que sofre influência de múltiplos fatores, tais como a ingestão proteica. Desta forma, o aumento da creatinina pode refletir melhora no estado nutricional, mesmo que seja um método pouco sensível, pois é afetado também pelo uso de medicamentos, dentre outras possibilidades ${ }^{(19)}$.

Na presente pesquisa, a terapia de substituição renal mais utilizada foi a contínua, possivelmente em função do melhor manejo de pacientes hemodinamicamente instáveis, pois oferece baixa remoção de solutos de forma contínua. A enfermagem participa ativamente das terapias de substituição renal, sendo a responsável pela parte técnica e relação do paciente com o ambiente, evidenciando a importância das enfermeiras frente às complicações, uma vez que a detecção e rápida intervenção é um diferencial importante na terapêutica instituída ${ }^{(11)}$.

Para o sucesso das terapias de substituição renal, ressalta-se a necessidade da manutenção do 
circuito e do cateter de diálise. Nesta pesquisa, parte dos pacientes foi submetida à anticoagulação regional (com citrato trissódico 4\%), considerado um método eficiente e seguro. Se associado ao controle metabólico adequado, ocorre bloqueio das etapas de coagulação dependentes do cálcio sérico, fazendo-se necessário o controle do cálcio iônico no circuito de hemodiálise. Em estudo ${ }^{(20)}$ que se propôs a avaliar o uso do citrato como anticoagulante em hemodiálise, não se detectou coagulação do sistema ou alteração importante do nível sérico de cálcio. O referido estudo apontou a salinização como alternativa recomendada aos pacientes com alto risco para sangramento ou na indisponibilidade do uso do citrato trissódico. O uso de heparina é amplamente empregado, todavia expõe o paciente à anticoagulação sistêmica e à trombocitopenia ${ }^{(18)}$.

Neste estudo, 68,92\% dos pacientes desenvolveram LRA em função do choque séptico. Esta condição, justamente com a sepse grave, constitui uma das principais causas de desenvolvimento de LRA em pacientes de terapia intensiva, com taxas de mortalidade muito superiores a pacientes que não desenvolveram LRA ${ }^{(21)}$.

O uso de drogas vasoativas constitui importante fator de risco para mortalidade em terapia intensiva, devido à instabilidade hemodinâmica dos pacientes e, em função do mecanismo de vasoconstrição, constitui-se como uma das possíveis causas de LRA ${ }^{(21)}$. Considera-se que aproximadamente a metade dos pacientes internados em UTI com diagnóstico de LRA em terapia dialítica evolui para óbito, devido ao aumento da gravidade clínica(15).

Embora tenha se identificado diferença estatisticamente significativa para os valores de creatinina sérica no término do tratamento nesta pesquisa, é preciso avaliar esta como marcador tardio de lesão renal aguda mediante variáveis não renais, tais como, idade, peso, massa muscular, ingestão proteica, dentre outros ${ }^{(22)}$.

Os fatores de risco de óbito reconhecidos na literatura em pacientes críticos com LRA incluem: idade avançada, prolongado tempo de internação, presença de comorbidades, oligúria, elevado nível de lactato, hipovolemia, acidose metabólica, sepse, politrauma, assim como o uso de fármacos vasoativos $^{(23)}$.

\section{- CONCLUSÕES}

Esta pesquisa contribuiu para a identificação do perfil clínico-demográfico dos pacientes internados na UTI que foram submetidos à terapia dialítica. A importância dada à gravidade mesmo no início do tratamento e o diagnóstico, bem como às intervenções diagnósticas e terapêuticas precoces, podem auxiliar no melhor reconhecimento da LRA e na proposição de tratamentos adequados.

O prognóstico reservado dos pacientes torna evidente a importância dos profissionais atuantes na UTI detectarem precocemente os sinais de LRA. Sugere-se, desta forma, a realização de estudos com maior número de paciente internados em UTI com LRA submetidos à terapia dialítica.

Identificar os sinais de LRA fornece subsídios para que o enfermeiro possa perceber prematuramente alterações de forma rápida, a fim de programar ações de enfermagem que minimizem complicações.

\section{- REFERÊNCIAS}

1. Fliser D, Laville M, Covic A, Fouque D, Vanholder R, Juillard L, et al. A European Renal Best Practice (ERBP) position statement on the Kidney Disease Improving Global Outcomes (KDIGO) clinical practice guidelines on acute kidney injury: part 1: definitions, conservative management and contrast-induced nephropathy. Nephrol Dial Transplant. 2012; 27(12): 4263-72.

2. Rolim LR, Frota NM, de Almeida NG, Barbosa IV, Melo EM. Clinical-epidemiological study of patients with acute renal failure. Rev enferm UFPE on line. 2012; 6(2): 317-23.

3. Cerqueira DP, Tavares JR, Machado RC. Fatores preditivos da insuficiência renal e algoritmo de controle e tratamento. Rev. Latino-Am. Enfermagem. 2014; 22(2): 211-7. 
4. Santos JCO, Mendonça MAO. Fatores predisponentes para lesão renal aguda em pacientes em estado crítico: revisão integrativa. Rev Soc Bras Clin Med. 2015; 13(1): 69-74.

5. dos Santos LL, Magro MCS. Ventilação mecânica e a lesão renal aguda em pacientes na unidade de terapia intensiva. Acta paul. enferm. 2015; 28(2): 146-51.

6. Patat CL, Stumm EMF, Kirchner RM, Guido LA, Barbosa DA. Análise da qualidade de vida de usuários em hemodiálises. Enferm Global. 2012; 11(3): 66-76.

7. Sampaio MC, Máximo CAG, Montenegro CM, Mota DM, Fernandes TR, Bianco ACM, et al. Comparação de critérios diagnósticos de insuficiência renal aguda em cirurgia cardíaca. Arq. Bras. Cardiol. 2013; 101 (1): 18-25.

8. Ponce D, Zorzenon CPF, dos Santos NY, Teixeira UA, Balbi AL. Injúria renal aguda em unidade de terapia intensiva: Estudo prospectivo sobre a incidência, fatores de risco e mortalidade. Rev. bras. ter. intensiva. 2011; 23(3): 321-6.

9. Santos ES, Marinho CMS. Principais causas de insuficiência renal aguda em unidades de terapia intensiva: intervenção de enfermagem. Rev. Enf. Ref. 2013; III(9): 181-9.

10. Triquez SL, Dallacosta FM. Perfil dos pacientes com insuficiência renal aguda na unidade de terapia intensiva e principais diagnósticos de enfermagem. Unoesc \& Ciência - ACBS. 2012; 3(2): 123-30.

11. de Oliveira APC, de Sousa AS, de Mendonça AEO, da Silva RAR. Intradialytic complications in patients with chronic renal failure submitted to hemodialysis: integrative review. J Nurs UFPE on line. 2013; 7(n.esp): 6639 -45.

12. Lima Neto PM, Lima EVNCL. Avaliação da função pulmonar e qualidade de vida de pacientes renais crônicos em fase de pré-transplante renal. Rev Pesq Saúde. 2013; 14(3): 141-4.

13. Soares M, Lobo SMA, Torelly AP, Mello PVC, Silva U, Teles JMM, et al. Outcomes of cancer patients admitted to Brazilian intensive care units with severe acute kidney injury. Rev. bras. ter. intensiva. 2010; 22(3): 236-44.

14. de Barros LCN, Silveira FS, Silveira MS, Morais TC, Nunes MAP, Bastos KA. Insuficiência renal aguda em pacientes internados por insuficiência cardíaca descompensada - Reincade. J. Bras. Nefrol. 2012; 34(2): 122-9.

15. Li PKT, Burdmann EA, Mehta RL. Injúria Renal Aguda: um alerta global. J. Bras. Nefrol. 2013; 35(1): 1-5.

16. Nunes TF, Brunetta DM, Leal CM, Pisi PCB, Roriz-Filho JS. Insuficiência renal aguda. Medicina (Ribeirão Preto). 2010; 43(3): 272-82.

17. Monedero P, García-Fernández N, Pérez-Valdivieso JR, Vives M, Lavilla J. Insuficiencia renal aguda. Rev Esp Anestesiol Reanim. 2011; 58(6): 365-74.

18. Lopes AA, Bragg-Gresham JL, Elder SJ, Ginsberg N, Goodkin DA, Pifer T, et al. Independent and joint associations of nutritional status indicators with mortality risk among chronic hemodialysis patients in the Dialysis Outcomes and Practice Patterns Study (DOPPS). J Ren Nutr. 2010; 20(4): 224-34.

19. Bueno CS, Frizzo MN. Anemia na doença renal crônica em hospital da região noroeste do estado do Rio Grande do Sul. J. Bras. Nefrol. 2014; 36(3): 304-14.

20. Amorim ÂMV, de Deus RB, Rodrigues FSM, Malagutti W, Ferraz RRN. Effectiveness of citrate as anticoagulant in continuos veno-venous hemodialysis with 60 hours cycle in intensive care unit. ConScientiae Saúde. 2010; 9(2): 187-93.

21. Okamoto TY, Dias JCY, Taguti P, Sacon MF, Kauss IAM, Carrilho CMDM, et al. Insuficiência renal aguda em pacientes com sepse grave: fatores prognósticos. Sci. Med. 2012; 22(3): 138-41.

22. Peres LAB, Cunha Júnior AD, Schäfer AJ, da Silva AL, Gaspar AD, Scarpari DF, et al. Biomarkers of acute kidney injury. J. Bras. Nefrol. 2013; 35(3): 229-36.

23. Druml W, Metnitz B, Schaden E, Bauer P, Metnitz PG.. Impact of body mass on incidence and prognosis of acute kidney injury requiring renal replacement therapy. Intensive Care Med. 2010; 36(7): 1221-8. 